\title{
Compound lasing fiber optic ring resonators for sensor sensitivity enhancement
}

\author{
Sergio Rota-Rodrigo, Miguel González-Herráez, and Manuel López-Amo Senior Member, IEEE
}

\begin{abstract}
In this work, a new method for increasing simultaneously the sensitivity and interrogation stability of fiberoptic intensity sensors is presented. The configuration is based on a double-coupler ring resonator. On one side, the attenuation sensitivity of the sensor is enhanced by placing it inside a ring resonator. On the other side, the stability of the interrogation method is widely improved by creating an external lasing ring resonator with matched spectral response. The combination of the wavelength selective response of the ring resonator and optical amplification from Er-doped fiber amplifier is used to create different fiber lasers. The resulting lasers present an impressive sensitivity enhancement and a dynamic range increment up to $15 \mathrm{~dB}$. Simultaneously, the instability of the system is reduced to $\pm 0.05 \mathrm{~dB}$. In comparison with systems based on tunable laser interrogation, our method is $>100$ times more stable and also simpler, as no wavelength tunable elements are required.
\end{abstract}

Index Terms - Optical fiber laser, Fiber optic ring resonator, Optical fiber sensor, Optical amplification.

\section{INTRODUCTION}

$\mathrm{O}$ PTICAL ring resonators (ORRs) have received increased attention in recent years due to their interesting optical properties. ORRs are structures with an optical feedback which provides them a periodical response in frequency. For this reason ORRs are used for communications as well as for sensors.

In the area of optical fiber communications, ORRs have been used for different applications. For example, they have been used to increase the in-band extinction ratio in add-drop filters [1] or for creating an optical wavelength detector based on an electrically controlled tunable micro-ring [2].

Inserting a gain element in the cavity, ORRs can be used to generate fiber ring lasers for telecommunications and for sensing applications. In [3] L.G. Yang et al. used a micro-ring resonator as filter for achieving the wavelength selection in an erbium doped fiber ring laser (EDFRL). Ring fiber lasers have

Manuscript received $\mathrm{XX}, \mathrm{XXX}$. The authors would like to acknowledge the financial support from the Spanish Ministerio de Educación y Ciencia through projects TEC2010-20224-C02-01, TEC2013-47264-C2-2-R, TEC2012-37958-C02-01 and Innocampus; from the European FEDER funding, ECOAL-MGT SUDOE project and from the European Research Council through Starting Grant U-FINE (Grant No. 307441).

S. Rota-Rodrigo and M. López-Amo are with the Department of Electronic and Electronic Engineering, Universidad Pública de Navarra, Campus Arrosadia s/n E-31006 Pamplona, Spain. (corresponding author e-mail: Sergio.rota@unavarra.es)

M. González-Herráez is with the Departamento de Electrónica, Universidad de Alcalá, Edificio Politécnico, Madrid 28871, Spain. been also been used for sensor interrogation for a long time, mostly in their multimode version [4]. The ORR can be also used to achieve a single longitudinal mode (SLM) operation laser like in [5] where, a combination of different ORR lengths were used for this purpose.

In addition, the sensors based on ORR can be classified in different groups according to their implementation technology, interrogation method, and topology.

In relation to the ORR implementation technology, two main groups can be distinguished; fiber optic ring resonators (FORRs) and integrated optic ring resonators (IORRs). The period of the ORR frequency response is inversely proportional to its length, for this reason IORRs will have a high wavelength separation between resonances in comparison with FORRs. IORR sensors are mostly used for monitoring the resonance wavelength shift as a consequence of the refractive index variations in presence of e.g. chemical substances $[6,7]$. However the IORR size makes it unsuitable for measuring physical parameters such as strain or displacement.

On the other hand, FORRs are used to measure physical as well as chemical parameters. For instance, in [8] C.Yu et al. presented a temperature sensor by using the nonlinear phase shift accumulated in the resonator for enhancing the phase difference between the two arms of a Mach-Zhender interferometer. In [9], L. Zhang et al. achieved a strain sensor using Vernier spectroscopy of a FORR's frequency comb. As IORRs, FORRs are sometimes also used for chemical analysis, as can be seen in [10]. FORRs are interesting structures in combination with intensiometric sensors. In particular, FORRs allow a substantial increase in the absorption/loss sensitivity of the inserted sensors into the ring, due to the multiple recirculations of light through the sensors. This enhanced sensitivity manifests especially in the resonant peaks that appear in the spectral response of the resonator, which display an enhanced sensitivity to the intracavity loss.

Interrogating these resonant peaks is nevertheless challenging. The length of the FORR is usually more than 1 meter, so the separation between resonant wavelength peaks is less than $2 \mathrm{pm}$. For this reason, to measure the spectral response of the structure it becomes necessary the use of a high resolution and precise spectral analysis which normally leads to undesirably expensive systems In systems based on simple amplitude detection, ultra-stable and ultra-narrow lasers are needed. Most often, systems based on tunable lasers are used. As the FORR length is affected by ambient factors,

Copyright (c) 2013 IEEE. Personal use of this material is permitted. However, permission to use this material for any other purposes must be obtained from the IEEE by sending a request to pubs-permissions @ieee.org. 
any interrogation method used usually leads to a considerable instability of the measurements as we show below. In order to avoid this instability, a sweep interrogation with a tunable laser can be carried out or, alternatively, an optical vector analyzer can be used [11]. All these options render the interrogation system substantially more expensive. Lower cost alternatives consist of using a time domain detection scheme, such as the based on the cavity ring-down (CRD) technique [12] that analyzes the light intensity decaying of an input pulse inside the cavity, or those based on the detection of wavelength variations [9]. However, cost in this case is shifted to the electronic elements allowing high-speed temporal gating and acquisition of the pulses (in the CRD technique) or in providing a fine electronic control of the resonator. Turning the resonator into a laser cavity seems a convenient way to simplify the interrogation method and increase the stability of the measurement.

With respect to the lasing ORR topology, it can be distinguished the configurations in which the system is formed by a unique ring that contains, at the same time, the amplification and the sensor [13], and the topologies in which the sensor is placed in an independent ring. In this last case the most common configuration is based on a $2 \times 2$ coupler that uses a port as an input, another one as the output, and the remaining two ports are connected forming the ring. In [11] this configuration employing a70:30 coupler was used to measure the variations in the amplitude of the resonance produced by loss inside the ring. In [14] this configuration was used to generate a new gyroscope that reduced the source cost and the noise level.

The sensor system presented in this paper is based on a compound ring resonator, by using a double coupler configuration. This ORR structure presents transmitted and reflected signals which can be used for sensing. This is an important point since the transmitted and reflected signals show complementary responses [15]. In combination with a gain medium providing laser action, these complementary responses can be used to obtain a stable and cost-effective interrogation of the ORR. Overall, our method allows increasing the sensitivity in intensity sensor measurements while keeping good output intensity stability. The FORR is formed by a double coupler configuration, whose transmitted and reflected signals show complementary responses. This way, one of the outputs provides a sensitive response to losses in the ring and the other provides a matched spectrum to interrogate it. The "self-interrogator" laser was obtained by the combination of the FORR reflected signal with a gain medium. With this configuration the instability of the system is reduced in comparison to the use of external interrogators and also, the active equipment is reduced to an optical amplifier as gain medium and a semiconductor detector or a power meter as the intensity measurement system.

\section{RING RESONATOR CHARACTERIZATION}

The objective of this study is to obtain a laser-sensor system based on a resonant ring allowing an increase of both the sensitivity and the stability. In this section we perform a theoretical and experimental study of the ring resonator in which our sensor is based.

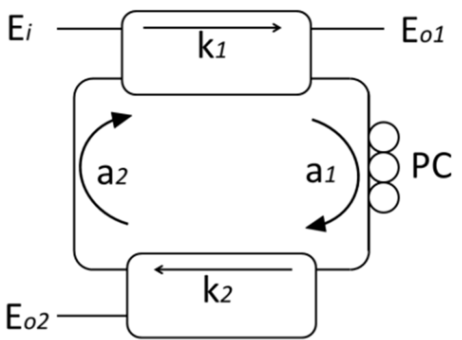

Fig.1 Compound fiber-based ring resonator.

As mentioned before, the ORR structure used in this paper uses two couplers forming a compound resonator. The upper coupler provides a sensitive response to the losses in the ring while the lower coupler shoes a complementary response to the upper one as shown below.

Considering the structure depicted in Fig. $1, E_{\mathrm{o} 1}$ and $E_{\mathrm{o} 2}$, are the transmitted and reflected signals of the FORR, respectively, and will be given by [11];

$$
\begin{gathered}
E_{o 1}=E_{i} \frac{k_{1} \exp \left(\frac{i w n L}{c}\right)-k_{2} a_{1} a_{2}}{\exp \left(\frac{i w n L}{c}\right)-k_{1} k_{2} a_{1} a_{2}} \\
E_{o 2}=\left(E_{i}-E_{o 1}\right)\left(1-k_{2}\right) a_{1}
\end{gathered}
$$

Where, $E_{\mathrm{i}}$ is the input signal, $k_{\mathrm{i}}$ and $a_{\mathrm{i}}$, are the coupling ratios and the attenuations in the resonator, respectively, and $L$ the total length of the ring [15].

As it is visible, the response of the ring is periodic in frequency, with resonant peaks spaced by a quantity $c / n L$. These resonances manifest as spectral dips in the power transmission response of $E_{\mathrm{o} 1}$. Under resonant conditions, the recirculation of power in the cavity is maximized, and the photon lifetime in the cavity grows significantly. In these resonant conditions, the sensitivity to small absorption/attenuation changes in the ring can be maximized, a fact that has been often used in spectroscopic measurements, as in cavity ring-down spectroscopy systems. However, it has been not often been used to enhance the sensitivity of conventional fiber-optic intensity sensors. The main reason is that it requires the interrogation of the spectral dip in the center of the resonance, which, as it will be seen later, requires precise and stable wavelength scanning lasers. We can now turn our attention to how much can the sensitivity be improved in this kind of setup in comparison with the direct readout of the intensity sensor. To evaluate this, we need to obtain the power transmission coefficient of the ring in the center of the dip, which can be written as [11]:

$$
T=\frac{\left|k_{1}-k_{2} a_{1} a_{2}\right|^{2}}{\left|1-k_{1} k_{2} a_{1} a_{2}\right|^{2}}
$$

The sensitivity to absorption or losses in the resonator can be evaluated by computing $d T / d a_{\mathrm{i}}$. The resulting expression is:

$$
\frac{d T}{d a_{i}} \propto\left|1-k_{1} k_{2} a_{1} a_{2}\right|^{-3}
$$


As it can be seen, higher values of $\mathrm{k}$ result in higher values of sensitivity. The sensitivity of the system is higher as we approach higher Q factors (as $k_{\mathrm{i}}$ and $a_{\mathrm{i}}$ approach 1 ). However this also leads to more challenging interrogation systems as the operation of the cavity becomes more and more narrowband. Hence a proper trade-off has to be found in these values. An initial study of the spectral response of the structure was carried out using $90 \%$ couplers $\left(k_{1}=k_{2}=0.9\right)$. The remaining values of the experimental setup were $L=2.14 \mathrm{~m}$ and, $a_{1}=a_{2}=0.05 \mathrm{~dB}$ due to the splices between the couplers. In an ORR, the resonance intensity depends on the light polarization. Therefore, in order to optimize the system response, a polarization control (PC) was included, and was set to maximize the resonance in all cases.

For the resonator frequency response characterization, a tunable laser source (Santec TLS-210) and a photo-detector (New Focus 1811) in combination with an oscilloscope (Agilent 54622A) were used as input and output, respectively. Within the TLS a wavelength sweep was generated and fed into the ORR. The output was measured in the time domain using the oscilloscope, and subsequently converted to frequency [11]. Fig. 2 shows the comparison of the experimental and theoretical simulation results.
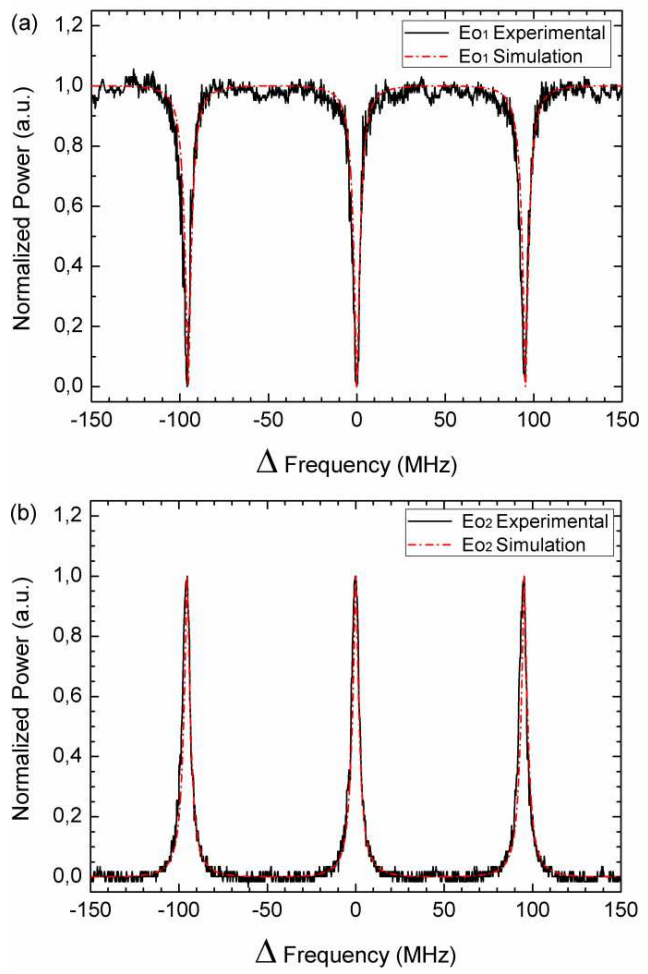

Fig. 2 Experimental and simulated normalized spectra of the resonator for $E_{\mathrm{o} 1}$ (a), and $E_{\mathrm{o} 2}$ (b).

As might be expected, the resonator spectra show resonances with a periodicity given by $c / n L$. In this case, the peaks separation was about $95 \mathrm{MHz}$, which is in good agreement with the expected value for $L=2.14 \mathrm{~m}$.

\section{ELECTION OF THE INTERROGATION METHOD}

Once the ring resonator response was characterized, the next step was to determine an interrogation method to measure the loss introduced by a sensor element (S) inside the ring.

The first configuration selected to carry out the characterization of the sensing ring consists on the use of a tunable laser source (TLS Agilent 81600B) as light source. The laser was operated at $1550 \mathrm{~nm}$, with a linewidth of 100 $\mathrm{KHz}$ and an instability of $\pm 1 \mathrm{pm}$ along 24 hours. As it is shown in Fig. 3, a power meter (Ando Aq2140) was used for the detection.

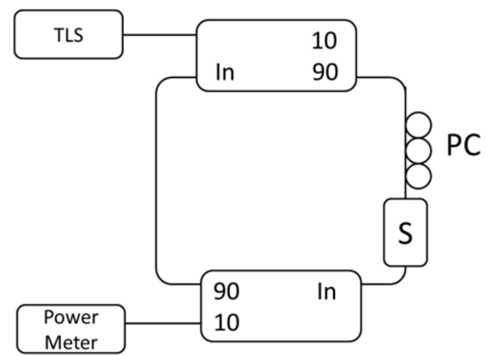

Fig. 3 Experimental setup of the TLS interrogation method.

In order to characterize the reliability of this configuration, a power instability measurement was carried out without attenuation $(\mathrm{S}=0 \mathrm{~dB})$. The measurement was done along 30 minutes, taking a sample each 10 seconds. Figure 4 shows the high instability behavior that made this interrogation method unsuitable for the vast majority of intensity sensor applications. The problem of this configuration is due to the transfer function instability of the ring in combination with the TLS wavelength instability. The separation of peaks in the transfer function is very narrow so small TLS wavelength variations cause a substantial change of the detected output power.

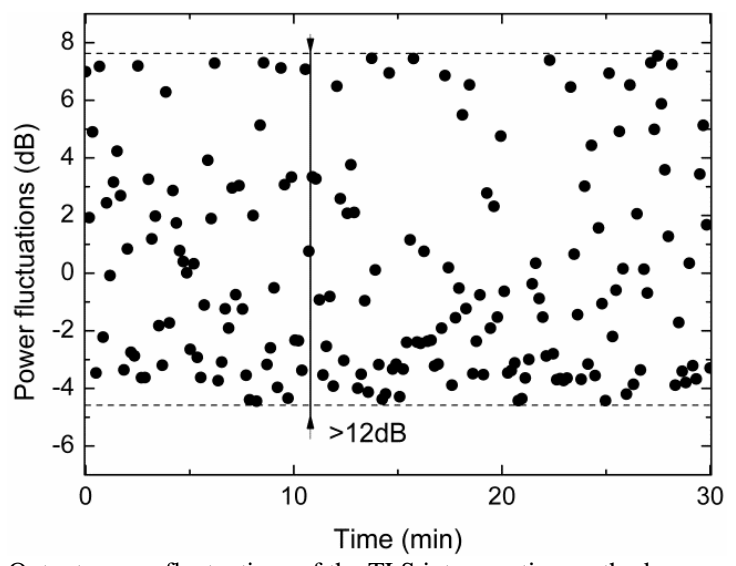

Fig. 4 Output power fluctuations of the TLS interrogation method.

The proposed solution was the use of the $E_{\mathrm{o} 2}$ output of the ring as seed, for generating a fiber ring laser, this way the structure itself behaves as the interrogator of the sensor. Any ambient variation leading to changes in the transmitted signal is immediately manifested in the reflected response. Hence, the "self-interrogation" of the structure seems a very convenient way to improve the stability of the measurement 
method. The actual configuration used is shown in Fig. 5. The gain medium is an Erbium doped fiber amplifier (EDFA: EFA-P21 from MPB Technologies). For the laser wavelength selection, a filter at $1533.9 \mathrm{~nm}$ with a BW of $0.1 \mathrm{~nm}$ was used. The polarization control was used to optimize the laser output power. Note that the lasing cavity in Fig. 5 is much longer than the ORR cavity, so effectively the spectral response of the obtained laser turns out to be approximately that of $E_{\mathrm{o} 2}$.

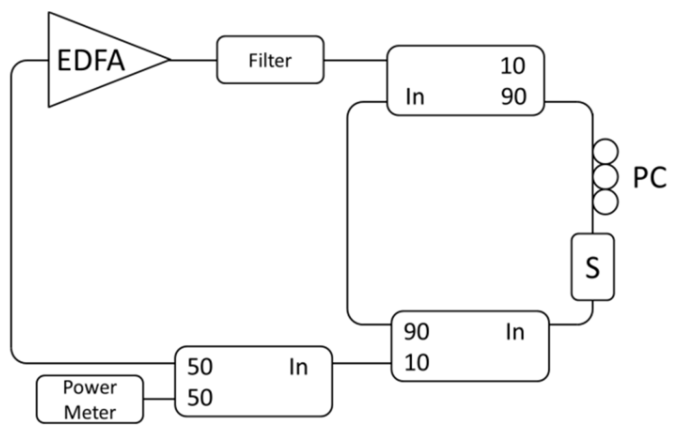

Fig. 5 Experimental setup of the EDFRL interrogation method.

As in the previous setup, an instability characterization was carried out with the sensor tuned at $0 \mathrm{~dB}$ attenuation, obtaining, as shown in Fig. 6, an instability lower than $0.1 \mathrm{~dB}$.

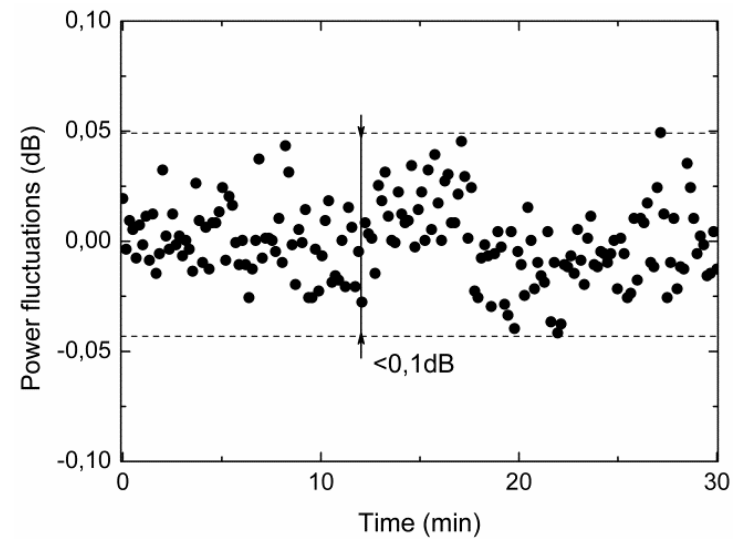

Fig. 6 Output power fluctuations of the EDFRL interrogation method.

Taking into account the results obtained, the selected method for the sensor interrogation was this Erbium doped fiber laser (EDFRL).

\section{CHARACTERIZATION OF THE EDFRL SENSOR SYSTEM}

Once the interrogation method has been chosen, the next step was the characterization of the system through the use of a variable attenuator (VA), simulating an intensity sensor element. For this purpose a voltage controlled VA was used in order to increase the precision and the resolution of the characterization.

Fig. 7 shows the proposed schematic in which there are two positions, A and B, where the sensor can be located. Placing alternatively the sensor in these two positions we can compare the behavior of a sensor inside the ring (B) and outside it (A). Furthermore, by disconnecting the internal ORR (dash line in
Fig. 7) the sensor can be compared with a simple ring configuration. The proposed system shows strong improvements in sensitivity compared to the open-loop response of the sensor and also in comparison with the conventional lasing ring resonator (see Fig. 8). A series of measurements varying the applied voltage of the VA from 0 to $4 \mathrm{~V}$ with steps of $10 \mathrm{mV}$ was carried out.

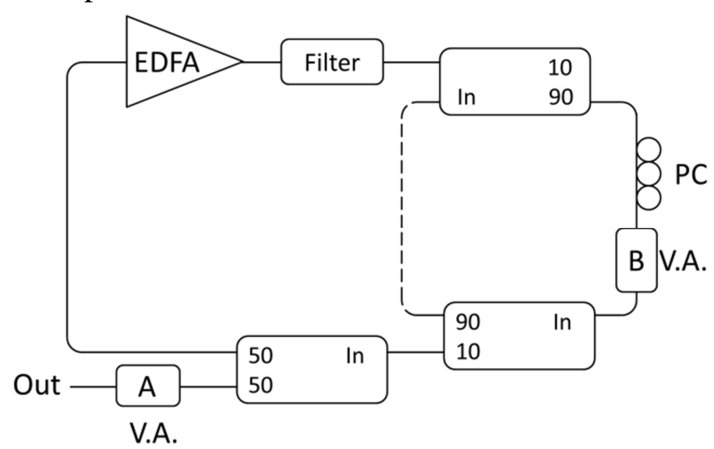

Fig. 7 Experimental setup of the EDFRL sensor system.

As can be seen in Fig. 8, when the VA was located inside the ring, the dynamic range of the system can be increased up to $15 \mathrm{~dB}$ in the operation range. The attenuation operation range was defined from $0 \mathrm{~dB}(0 \mathrm{~V})$ to $7.5 \mathrm{~dB}(3.25 \mathrm{~V})$, where the loss of the cavity finally prevents the laser condition.

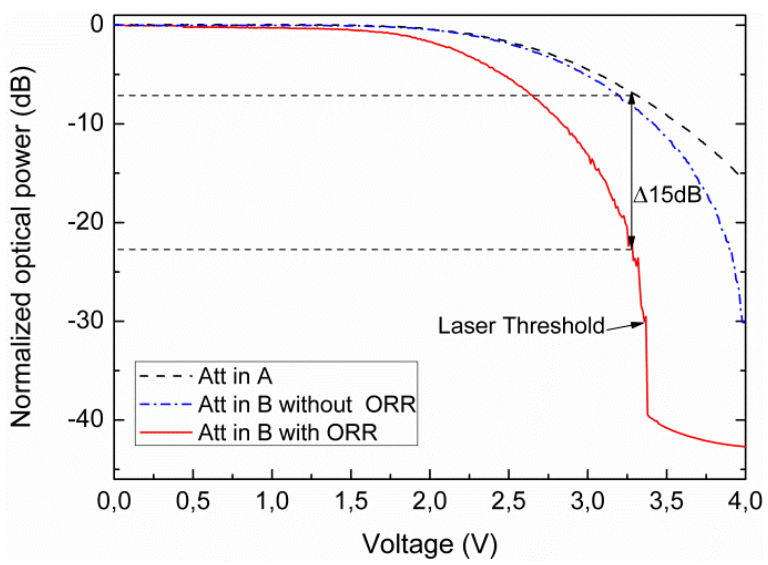

Fig. 8 Output power response against the voltage applied to VA located in position A (dash), position B (continuous) and position B without ORR (dashdotted).

Also a study of the output power instability following the VA attenuation inside the ring was performed. The system was tested for 11 different voltage values of the VA and each measurement was carried out along 30 minutes, using an acquisition time of 10 seconds.

The obtained results, Fig. 9, show an instability close to 0.1 $\mathrm{dB}$ for the whole VA range up to $2.1 \mathrm{~V}$. From this value onwards, the instability increases up to a maximum of $0.6 \mathrm{~dB}$, when a voltage of $3 \mathrm{~V}$ is applied to the attenuator. This is again justified by the close operation of the system to the laser threshold. 


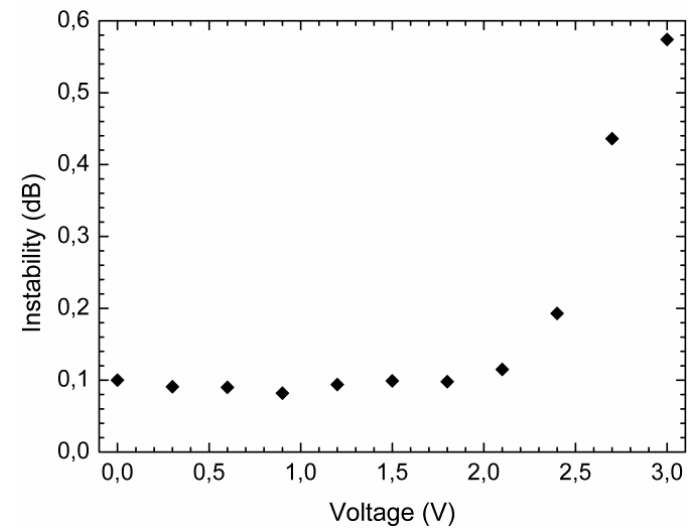

Fig. 9 Output power instability of the structure against the voltage applied to VA.

As stated at section 2, the sensitivity of the system is higher for higher $k$ values (see eq. 4 ). In order to confirm this, an experimental study for $k_{1}=0.5$ and $k_{1}=0.9$ coupling ratios, (recirculating inside the ring the $50 \%$ and $90 \%$ of the optical power, respectively), was carried out. Figure 10 shows that in both cases the achieved output power of the lasing system was similar, but with the higher coupling ratio $\left(k_{1}=0.9\right)$ the sensitivity increases, as expected from the previous theoretical arguments.

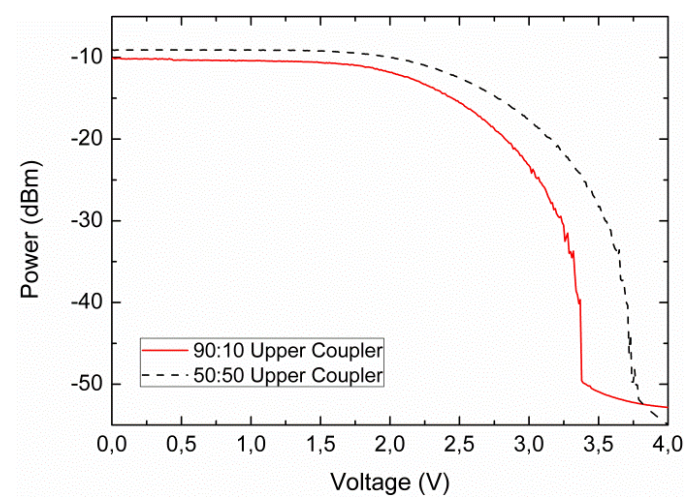

Fig. 10 Output power response against the voltage applied to a VA for two different coupling ratios of the upper coupler.

\section{STUDY OF THE APPLICATION OF THE EDFRL SENSOR SYSTEM FOR DISPLACEMENT MEASUREMENT}

In this section a practical case for displacement measurements based on a bending sensor was investigated. It is known that the sensors based on bending an optical fiber have a non-linear response due to the cladding-modes coupling effect [16]. Therefore, three different spectral configurations of our EDFRL sensor system were studied in order to determine the best option for this kind of sensors.

The experimental setup for the EDFRL sensor system is shown in Fig. 11. The difference of each configuration consists on the different filters used; no filter for a wide-band interrogation, a filter with a $\mathrm{BW}=0,1 \mathrm{~nm}$ for achieving a multimode laser interrogation and the combination of this filter with a phase-shifted fiber Bragg grating (PSFBG) to obtain a single-longitudinal mode interrogation, corresponding with sections $5 \mathrm{~A}, 5 \mathrm{~B}$ and $5 \mathrm{C}$ respectively.
The gain medium was again the previously utilized EDFA (EFA-P21) and the output power detection was made through a power meter (AQ2140).

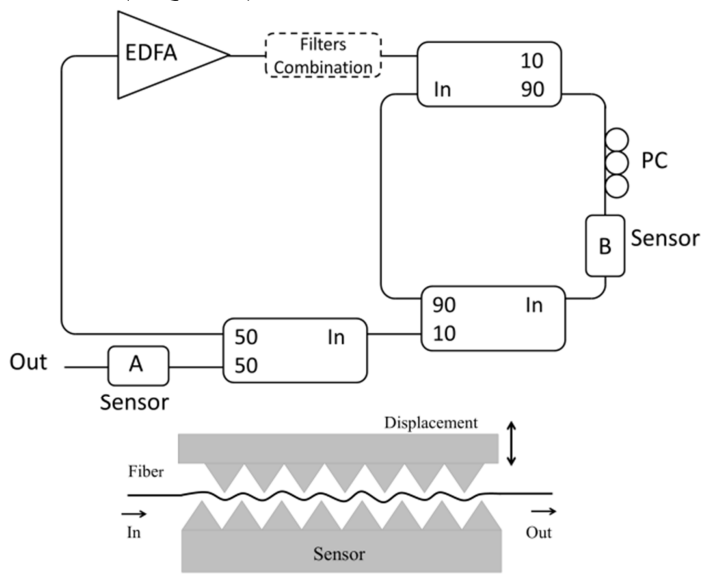

Fig. 11 Experimental setup of EDFRLs for the displacement sensor interrogation system and, schematic of the displacement sensor based on bending.

The characterization of each scheme was conducted through a precision mechanic attenuator based on stress-inducing plates, similar to the one used in [17]. All measurements were carried out in the same way, ranging from 0 to $1000 \mu \mathrm{m}$ with a step of $4.27 \mu \mathrm{m}$. Before each measurement, the polarization was optimized using the PC.

In the sub-sections below, we present the experimental results for each configuration, in each case analyzing the performance in terms of the next different parameters: maximum output power, measurement range, dynamic range increase, instability and cladding-modes coupling behavior. The dynamic range increment was in all cases measured at $400 \mu \mathrm{m}$ because this value corresponds to the minimum displacement range of section 5. The instability was also measured at $400 \mu \mathrm{m}$ along 30 minutes, taking a sample each 10 seconds.

\section{A. Wideband Ring-Based System}

In the first case, no additional filtering inside the ring laser was used, so the interrogation method was based on a wideband laser. Figure 12 shows the measured results for the sensor at positions $\mathrm{A}$ and $\mathrm{B}$.

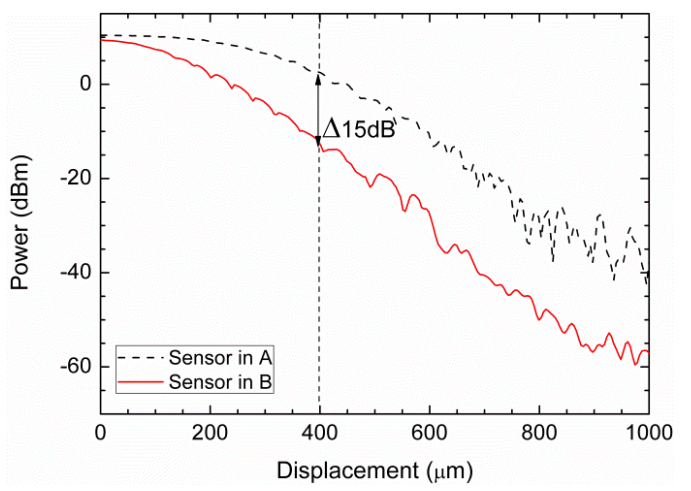

Fig. 12 Output power response versus the displacement applied to the sensor, when located in position A (dotted) and B (continuous). 
As seen above, when the sensor is located inside the ring, a dynamic range increment of $15 \mathrm{~dB}$ at $400 \mu \mathrm{m}$ was achieved. This configuration allows to measure up to $1000 \mu \mathrm{m}$.

The cladding modes coupling effect generates a ripple on the sensor response. In order to quantify this effect the output power response was fitted up to $400 \mu \mathrm{m}$ by a second-order polynomial. The ripple was quantified trough the coefficient of determination $\left(\mathrm{R}^{2}\right)$, which is defined as the square of the sample correlation coefficient between the measured values and their predicted values [18]. For the wide band ring based system a $\mathrm{R}^{2}$ of 0.99711 and an instability of $0.21 \mathrm{~dB}$ were measured.

\section{B. Multi-Longitudinal Mode Fiber Ring Laser-Based System}

In this case, a multimode EDFRL was created. Fig. 13 shows the optical spectrum of the laser and the filter transmission (inset) measured with a high resolution $(0.08 \mathrm{pm})$ optical spectrum analyzer (BOSA OPT 100).

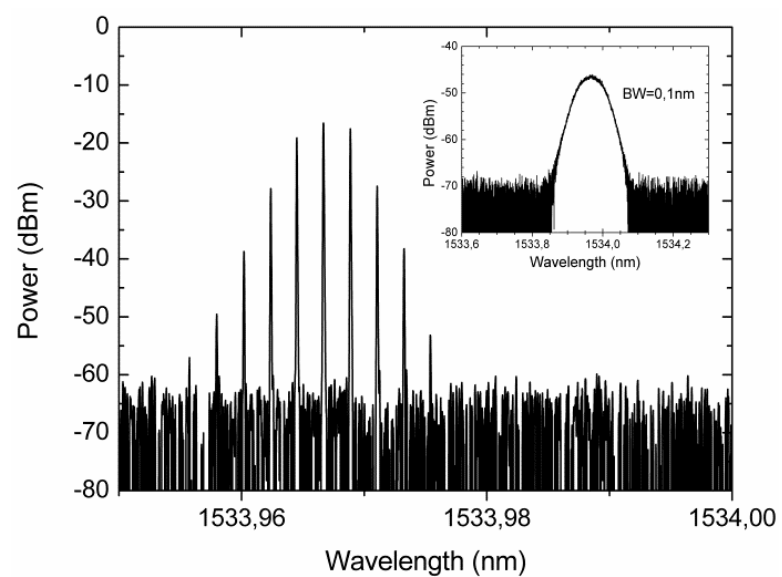

Fig. 13 Optical spectrum of the multi longitudinal mode EDFRL and the filter transmission (inset) measured with a BOSA.

The characterization, Fig. 14, shows the same sensitivity increment at $400 \mu \mathrm{m}$, as in the previous configuration. Also a low cladding-modes coupling effect was appreciated. As in the previous case, a fit up to $400 \mu \mathrm{m}$ was carried out showing a $\mathrm{R}^{2}$ of 0.99775 .

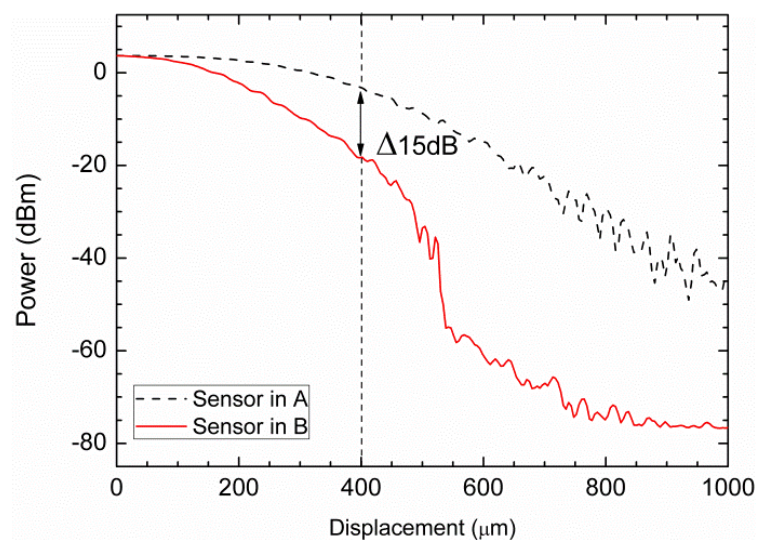

Fig. 14 Output power response in function of the sensor displacement in position A (dotted) and B (continuous).
Because this configuration is operating as an EDFRL, there was an attenuation threshold from which the laser condition was not satisfied, reducing the sensor range to $530 \mu \mathrm{m}$. Also, the system presented an instability of $0.34 \mathrm{~dB}$.

\section{Single Longitudinal-Mode Fiber Ring Laser-Based System}

In order to achieve a single longitudinal mode (SLM) operation EDFRL, a PSFBG was added to the configuration. Fig. 15 shows the optical spectra of the SLM laser and the PSFBG transmission (inset) measured with BOSA.

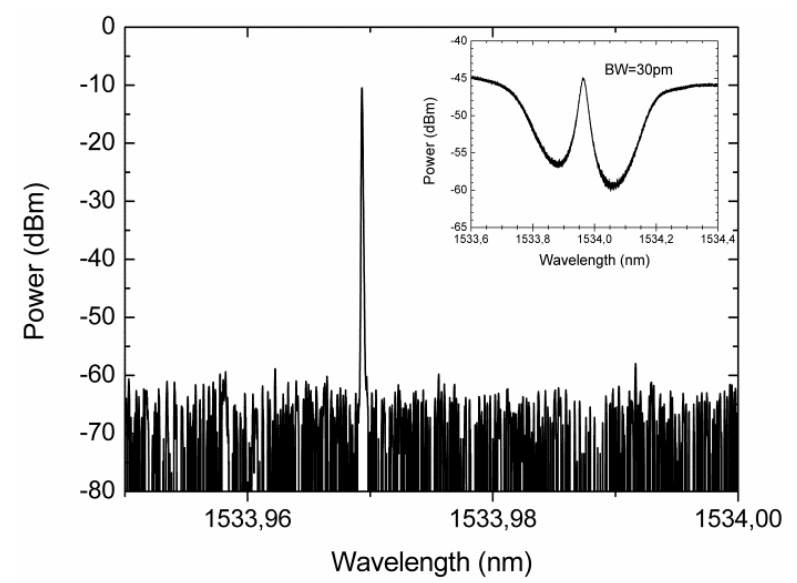

Fig. 15 Optical spectrum of the single longitudinal mode EDFRL and the filter transmission (inset) measured with a BOSA.

Fig. 16 shows that in this case there was a dynamic range increment of $18 \mathrm{~dB}$ at $400 \mu \mathrm{m}$ in comparison with the $15 \mathrm{~dB}$ achieved in sections 5A and 5B. However, since all the power was concentrated in a single longitudinal mode, the claddingmodes coupling effect was enhanced, showing a $\mathrm{R}^{2}$ of 0.98326 (lower than the previous cases). The sensor range was reduced to $400 \mu \mathrm{m}$ and the maximum output power was less than -3 $\mathrm{dBm}$. In this case the instability was increased up to $1.48 \mathrm{~dB}$. The high wavelength sensitivity of the PSBG to temperature variations in combination with the filter provokes this increment of the instability.

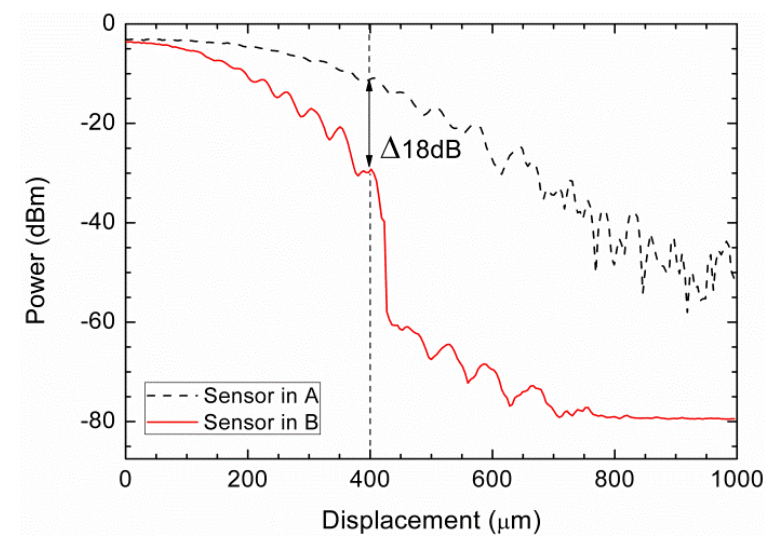

Fig. 16 Output power response in function of the sensor displacement measured at position A (dotted) and at position B (continuous). 


\section{Conclusions of the Intensity Bending Sensor Study}

To summarize the analysis carried out in section 5 , a table with the studied parameters of each configuration is presented (Table 1).

\begin{tabular}{|c|c|c|c|}
\hline & No-filter & $\begin{array}{l}\text { Filter } \\
0,1 \mathrm{~nm} \\
\end{array}$ & $\begin{array}{l}\text { Filter } 0,1 \mathrm{~nm} \\
+ \text { PSBG }\end{array}$ \\
\hline Sensor Range $(\mu \mathrm{m})$ & 1000 & 530 & 410 \\
\hline $\mathrm{P}_{\max }(\mathrm{dBm})$ & 9.5 & 3.7 & -3.5 \\
\hline $\begin{array}{l}\text { Dynamic Range Inc. } \\
@ 400 \mu \mathrm{m}(\mathrm{dB})\end{array}$ & 15 & 15 & 18 \\
\hline $\begin{array}{l}\mathrm{R}^{2} \text { of the Fit up to } \\
400 \mu \mathrm{m}\end{array}$ & 0.99711 & 0.99775 & 0.98326 \\
\hline $\begin{array}{l}\text { Instability @ } 400 \\
\mu \mathrm{m}(\mathrm{dB})\end{array}$ & 0.21 & 0.34 & 1.48 \\
\hline
\end{tabular}

Table 1 Critical parameters comparison of the different sensor configurations.

For this kind of sensors, the cladding-modes coupling is a critical point since it could cause errors in the measurements. Because of that, the system based on the $0.1 \mathrm{~nm}$ filter in combination with the PSFBG appears clearly as the worst option with the lower $\mathrm{R}^{2}$.

On the other hand the non-filtered and $0.1 \mathrm{~nm}$ filtered systems show comparable dynamic range increments and instabilities, and also the lowest dependence to the claddingmodes coupling so, for medium displacement ranges, these options can be considered the best configurations for our micro-bending sensor system. However, the use of the filter allows selecting and locking the wavelength emission of the laser, that is an important issue if several rings were to be multiplexed.

\section{FINAL CONCLUSIONS}

To summarize, in this paper a new system for enhancing fiber-optic intensity sensor measurements, based on a resonant ring cavity, is presented. The main advantage of this method is the ability to increase the dynamic range of an intensity sensor up to $15 \mathrm{~dB}$, while keeping good stability values. The solution is based on the use of a compound lasing fiber-optic ring resonator, in which the intensity sensor is inserted. Because the reflected function of the ring-resonator is used to generate the EDFRL, the lasing system is perfectly matched to work as a "self-interrogator" of the sensor. The resulting system shows enhanced sensitivity and high stability, showing an output power instability lower than $0.1 \mathrm{~dB}$. This performance is orders of magnitude better than the one achieved using an external interrogation source that presents an instability of 12 $\mathrm{dB}$, as seen in the section 3. The proposed interrogation system is particularly suited for any kind of transmissive intensity sensors and only requires two active elements, an EDFA as gain medium and an optical power meter acting as detector.

In addition, the concept has been tested for the development of displacement sensors based on fiber optic bending. A study of 3 different topologies, based on an EDFRL, with different filters, were carried out in section 5 . As a result of this study it was concluded that laser configurations without additional filtering or using a filter of $0.1 \mathrm{~nm}$, showed the best results with a dynamic range increment higher than $15 \mathrm{~dB}$, and a comparatively low cladding-modes coupling effect.

\section{REFERENCES}

[1] M. A. Popović, T. Barwicz, M. R. Watts, P. T. Rakich, L. Socci, E. P. Ippen, F. X. Kärtner and H. I. Smith, "Multistage high-order microringresonator add-drop filters," Opt. Lett. Vol.31, pp. 2571-2573 ,2006.

[2] J. F. Tao, H. Cai, J. Wu, J. M. Tsai, Q. X. Zhang, J. T. Lin, and A. Q. Liu, "Optical wavelength signal detector via tunable micro-ring resonator for sensor applications," Proc. IEEE 26th International Conference on MEMS, pp.500-503,2013.

[3] L. G. Yang, C. H. Yeh, C. Y. Wong, C. W. Chow, F. G. Tseng, and H. K. Tsang, "Stable and wavelength-tunable silicon-micro-ring-resonator based erbium-doped fiber laser," Opt. Express 21, pp. 2869-2874,2013.

[4] E. Achaerandio, S. Jarabo, S. Abad, and M. Lopez-Amo, "New WDM amplified network for optical sensor multiplexing," IEEE Phot. Tech. Lett. Vol. 11, pp. 1644-1666, 1999.

[5] C. H. Yeh, T. T. Huang, H. C. Chien, C. H. Ko, and S. Chi, "Tunable Sband erbium-doped triple-ring laser with single-longitudinal-mode operation," Opt. Express, Vol. 15, pp. 382-386, 2007.

[6] V. M. N. Passaro, C. de Tullio, B. Troia, M. La Notte, G. Giannoccaro, and F. De Leonardis, "Recent Advances in Integrated Photonic Sensors," Sensors, Vol. 12, pp. 15558-15598, 2012.

[7] J. Saunders, M. A. Dreher, J. A. Barnes, C. M. Crudden, R. S. Brown, H. P. Loock, and D. X. Xu, "Detection of volatile organic compounds with functionalized long-period gratings and micro-ring resonators," Proc. in 2011 ICO International Conference on Information Photonics, pp.1-2, 2011.

[8] C. Yu, Y. Zhang, X. Zhang, K. Wang, C. Yao, P. Yuan, and Y. Guan, "Nested fiber ring resonator enhanced Mach-Zehnder interferometer for temperature sensing," Appl. Opt., Vol. 51, pp. 8873-8876, 2012.

[9] L. Zhang, P. Lu, L. Chen, C. Huang, D. Liu, and S. Jiang, "Optical fiber strain sensor using fiber resonator based on frequency comb Vernier spectroscopy," Opt. Lett. Vol. 37, pp. 2622-2624, 2012.

[10] Y. Guo and X. Fan, "Optofluidics in Bio-Chemical Analysis," Proc. SPIE, Vol.8212, 82120F, 2012.

[11] M. Bravo, X. Angulo-Vinuesa, S. Martin-Lopez, M. Lopez-Amo and M. Gonzalez-Herraez, "Slow-light and enhanced sensitivity in a displacement sensor using a lossy fiber-based ring resonator," Journal of Light. Technol, Vol. 31, pp 3752-3757, 2013.

[12] C. Wang, Sensors, "Fiber Loop Ringdown- a Time-Domain Sensing Thecnique for Multi-Function Fiber Otpic Sensor Platforms: Current Status and Design Perspectives," Vol. 9, pp. 7595-7621, 2009.

[13] J.M. Baptista, C. Correia, M.B. Marques, O. Frazão, “A Raman Laser Intensity Sensor Induced by the Cooperative Rayleigh Scattering in a Ring Configuration," Laser Phy., Vol. 21, pp. 928-930, 2011.

[14] S. Zhu, G. Huang, W. Fan, W. Liu, Y. Tang, Q. Mo, X. Hu, and Z. Li, "Double-coupler fiber resonator gyroscope," Proc. In 2010 Internacional Conference on Optoelectronics and Image, 2013.

[15] S. Rota-Rodrigo, M. González-Herráez and M. López-Amo, "Compound lasing fiber optic ring resonators for sensing," Proc. SPIE 9157, $23^{\text {rd }}$ Intenational Conference on Optical Fibre Sensors, 9157D7, 2014.

[16] M. Bravo and M. López-Amo, "Remote-Time Division Multiplexing of Bending Sensors Using a Broadband Light Source,"Journal of Sensors 2012, Article ID 154586, 2012.

[17] M. Bravo, A. M. R. Pinto, M. Lopez-Amo, J. Kobelke and K. Shuster, "High precision micro-displacement fiber sensor through a suspendedcore Sagnac interferometer," Opt. Lett. Vol. 37, pp. 202-204, 2012.

[18] N.R. Draper, H. Smith, Applied Regresion Analysis (John Wiley \& Son, 1981), chap 1. 